\title{
Modelagem de Objetos Inteligentes de Aprendizagem utilizando a metodologia MaSE
}

\author{
Júlia Marques Carvalho da Silva \\ Ricardo Azambuja Silveira \\ Universidade Federal de Santa Catarina, Campus Universitário, s/n ${ }^{\circ}$ \\ Caixa Postal, 467 - Florinanópolis - SC, Brasil \\ \{julia, silveira\}@inf.ufsc.br
}

Resumo. O presente trabalho apresenta a modelagem de um Sistema Multiagente para Objetos Inteligentes de Aprendizagem utilizando a metodologia para Engenharia de Sistemas Multiagentes, denominada MaSE (Multiagent System Engineering). Esta metodologia permite descrever desde uma especificação inicial do sistema até a sua implementação através da interligação entre seus diagramas.

Palavras-chave: Engenharia de Sistemas Multiagentes, Objetos Inteligentes de Aprendizagem, Objetos de Aprendizagem, AgentTool.

\section{Modeling Intelligent Learning Objects through Multiagent Systems Engineering}

Abstract: This paper presents a Multiagent Systems modeling to Intelligent Learning Objetcs using the Multiagent Systems Engineering (MaSE) methodology. This methodology allows to describe a whole process of multiagent system design, since the initial specification of goals, until agent class implementation, using a set of interrelated models.

Keywords: Multiagent Systems Engineering, Intelligent Learning Objects, Learning Objetcs, AgentTool. 


\section{Introdução}

Neste trabalho é apresentada a modelagem de uma abordagem proposta por Silveira et al. (2005), na qual objetos de aprendizagem são construídos com base no paradigma de agentes. A fundamentação tecnológica desta abordagem é constituída por uma integração entre tecnologias desenvolvidas para Objetos de Aprendizagem e para Sistemas Multiagentes (SMAs).

O conceito central apresentado é o de Objeto Inteligente de Aprendizagem, entidade que corresponde a um agente com a capacidade de gerar experiências de aprendizagem reutilizáveis, no mesmo sentido que os objetos de aprendizagem. Para tanto, é apresentada uma sociedade multiagente capaz de sustentar tal conceito.

Com base na arquitetura apresentada por Silveira et al. (2005), foi realizada a modelagem do SMA, utilizando como base conceitual a metodologia MaSE Multiagent Systems Engineering - (DeLoach e Wood, 2000). O MaSE permite ao projetista identificar o que deverá ser desenvolvido, ou seja, quais e como os agentes se comportarão na sociedade. A metodologia propõe uma seqüência de modelos que mapearão desde os objetivos gerais no qual se pretende atingir, até a definição dos casos de usos, papéis, classes e diálogos entre os agentes.

\section{Objetos Inteligentes de Aprendizagem}

Um Objeto de Aprendizagem é um pedaço de conteúdo de aprendizagem que pode ser aplicado ao aprendizado em diversos momentos, e em diferentes cursos e situações (Downes, 2001; Mohan e Brooks, 2003; e Sosteric e Hesemeier, 2002). Os objetos podem ter seus métodos invocados por algum Sistema Gerenciador de Aprendizagem (Learning Management Systems - LMS), contudo estão limitados a funcionar de uma forma específica.

Silveira et al. (2005), apresentam uma abordagem sobre os Objetos de Aprendizagem, agregando a tecnologia de Sistemas Multiagentes (SMAs), denominado Objetos Inteligentes de Aprendizagem (Intelligent Learning Objetcs - ILO). A utilização de SMAs ampliam as possibilidades aos ambientes de aprendizagem tradicionais, por apresentarem características como (Bradshaw, 1997):

- Um agente é um pedaço de software que trabalha de forma contínua e autônoma, dentro de um ambiente;

- Um agente pode inferir em um ambiente de forma flexível e inteligente, sem necessitar de intervenção humana; e,

- Um agente pode se comunicar com outros agentes através de troca de mensagens, usando Linguagens de Comunicação entre Agentes (Agent Communication Language - ACL).

As características acima citadas podem ser aplicadas ao contexto dos Objetos Inteligentes de Aprendizagem, da seguinte forma: um ILO é um pedaço de software, no qual se pode adaptar a partir de informações recebidas por outros agentes devido a sua capacidade de inferência, sem necessitar de intervenção externa.

Outra possibilidade interessante refere-se a capacidade de aprendizagem, na qual os agentes possuem. Logo, um objeto de aprendizagem com esta característica pode adquirir novos conhecimentos e comportamentos no decorrer de sua existência, através da interação com outros alunos e, até mesmo, com outros objetos de aprendizagem, tais como: adquirir novos materiais educacionais que possam auxiliar o aluno $\mathrm{e}$ complementar o ensino, adquirir informações sobre os alunos, como as suas preferências e estilos cognitivos, a fim de se adaptar a estes e, inclusive, aprender como 
se adaptar a elas; mudar seu conteúdo educacional no sentido de se adaptar ao estudante; entre outras.

\section{Sociedade de Agentes para o ILO}

A sociedade de agentes apresentada por Silveira et al (2005), ilustrada na Figura 1 , é composta por três tipos de agentes:

- Agente LMS: representa os sistemas de gerenciamento de aprendizagem, lidando com os aspectos administrativos e gerenciais que envolvem os ambientes de aprendizagem. Além disso, provê o acesso dos aprendizes aos ILOs, e fornece informações do aprendiz aos ILOs;

- Agente ILO: encapsula os objetos de aprendizagem, sendo capaz de gerar experiências de aprendizagem aos alunos; e,

- Agente ILOR (Intelligent Learning Object Repository): são abstrações dos sistemas de repositório de objetos de aprendizagem, armazenando dados sobre os ILOs, possibilitando a usuários ou agentes encontrá-los.

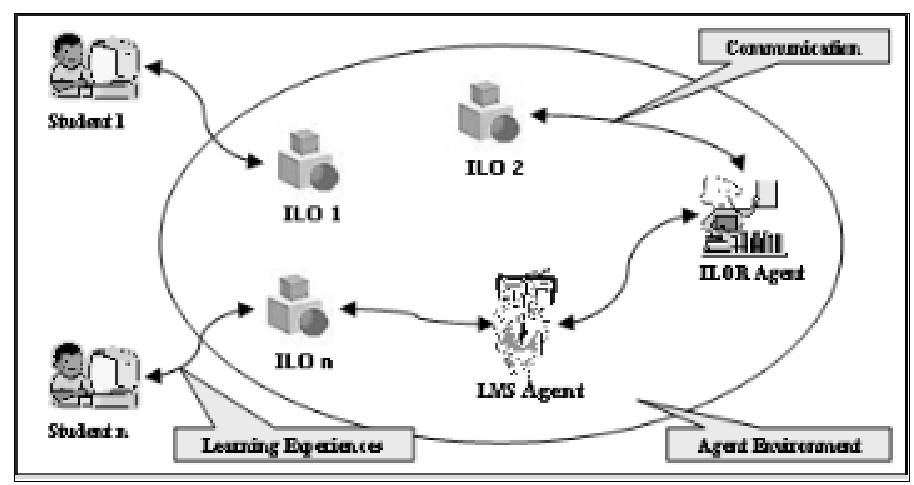

Figura 1. Sociedade de agentes proposta.

Inicialmente, os aprendizes interagem com o agente LMS a fim de obter experiências de aprendizagem. O agente LMS busca o ILO mais apropriado para a necessidade de aprendizagem do aprendiz e o invoca. O ILO é então responsável por gerar as experiências de aprendizagem ao aprendiz. Nesta tarefa, é possível comunicarse com o agente LMS e com outros agentes ILO a fim de promover uma experiência de aprendizagem mais rica. Toda comunicação é promovida pela troca de mensagens no padrão FIPA-ACL. O ambiente onde tais agentes habitam estão no padrão FIPA, o qual provê todo o mecanismo necessário para troca de mensagens entre os agentes.

\section{Modelando o Sistema Multiagente}

O Sistema Multiagente foi modelado através da metodologia proposta por DeLoach e Wood (2001), denominada MaSE. Ela permite o desenvolvimento de sistemas multiagentes com base nos princípios da Engenharia de Software. Para tanto, o processo de desenvolvimento é dividido em duas fases principais: a análise e a modelagem; onde cada um provê um conjunto de etapas a serem seguidas. A Figura 2 apresenta a metodologia MaSE. 


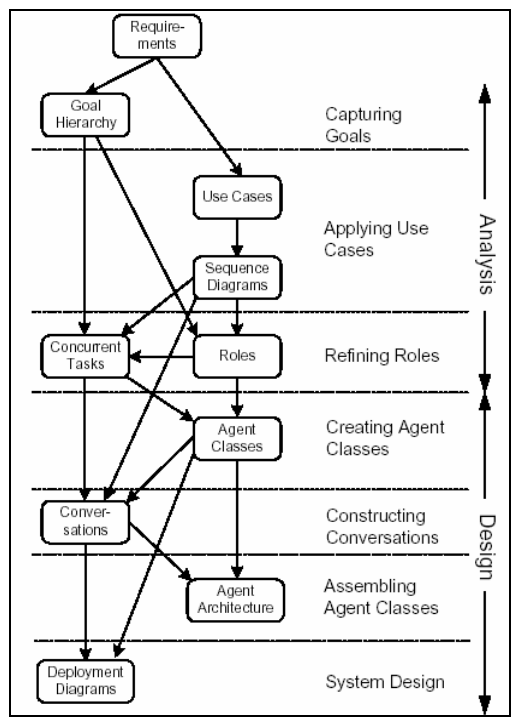

Figura 2. Diagrama de hierarquia de objetivos.

A fase de análise consiste nas seguintes etapas: capturar dos objetivos, estabelecer os casos de uso, e definir os papéis; já a fase de modelagem contempla as etapas: criar as classes de agentes, desenvolver os diálogos, reunir as classes dos agentes, desenvolver a estrutura do sistema.

A metodologia MaSe foi desenvolvida para ser aplicada de forma interativa. Espera-se que o desenvolver possa navegar entre as etapas em diversos momentos, acarretando em um modelo completo e consistente (DeLoach e Wood, 2001). Os modelos são transformados em diagramas que permite descrever os agentes, seus diálogos e estrutura interna, em diferentes níveis de detalhamento. Ainda, o desenvolvedor pode selecionar quais diagramas irá utilizar e em que ordem eles serão descritos. Ao final, obtém-se uma consistência entre os modelos do MaSE de forma a satisfazer os objetivos iniciais do sistema.

Na sequiência é apresentada a modelagem da sociedade de agentes proposta por Silveira et al (2005), utilizando a ferramenta agentTool, na qual implementa a metodologia MaSE através de seus diagramas.

\subsection{Capturando os objetivos}

A identificação dos objetivos do SMA visa conhecer as especificações do sistema e transformá-las em uma estrutura de conjunto de objetivos. Conforme a metodologia, é necessário conhecer qual é o objetivo principal do SMA, para então expandir em sub-objetivos os quais permitirão atingir o primeiro. No caso dos Objetos Inteligentes de Aprendizagem, o objetivo fundamental é proporcionar aos aprendizes experiências de aprendizagem. Para que isto seja possível, é necessário que as três entidades (ambiente, objeto e aluno) estejam aptos a realizar suas tarefas específicas, como, por exemplo, o ambiente deve ser capaz de receber uma solicitação de experiência de aprendizagem. Os objetivos identificados para este contexto são apresentados na Figura 2. 


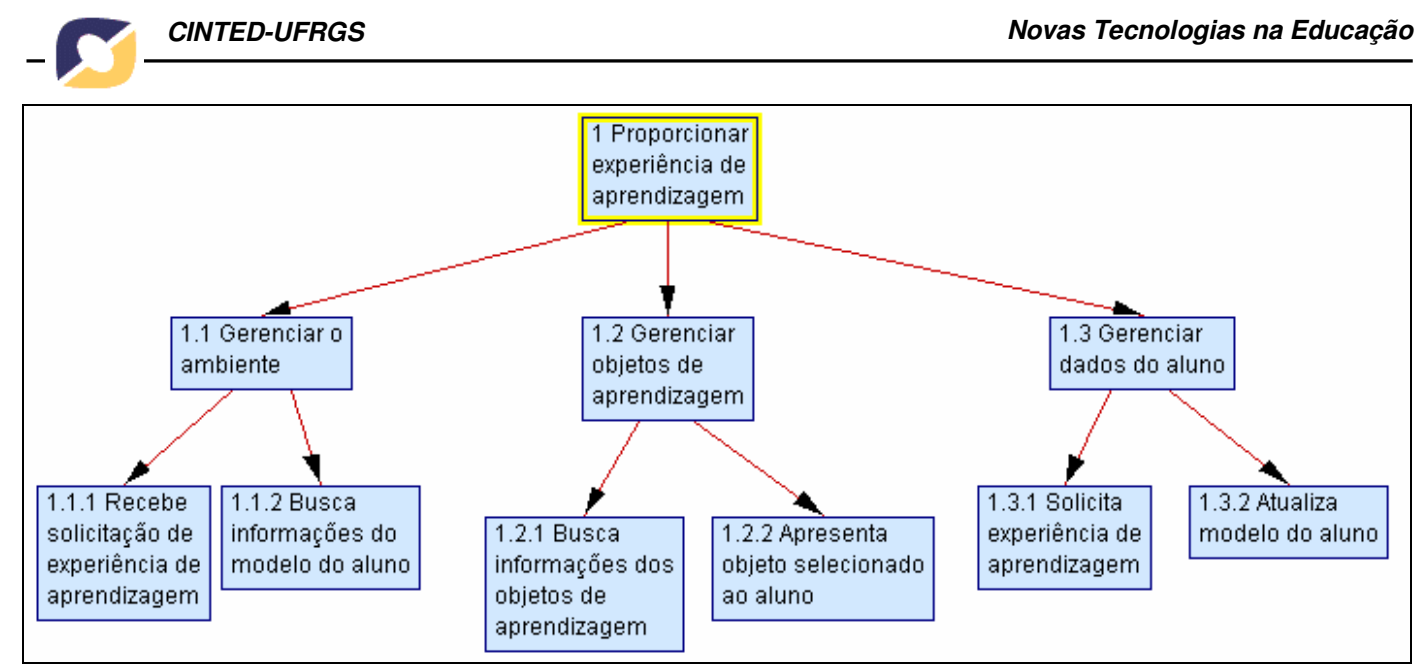

Figura 2. Diagrama de hierarquia de objetivos.

\subsection{Estabelecendo os papéis}

Esta etapa permite definir através de um diagrama os papéis (representados em retângulos), tarefas (representadas pelas elipses) e protocolos de comunicação (representados pelas setas) que o SMA deverá conter. No diagrama, todos os objetivos estabelecidos inicialmente devem ser vinculados a um papel, conforme é apresentado na Figura 3.

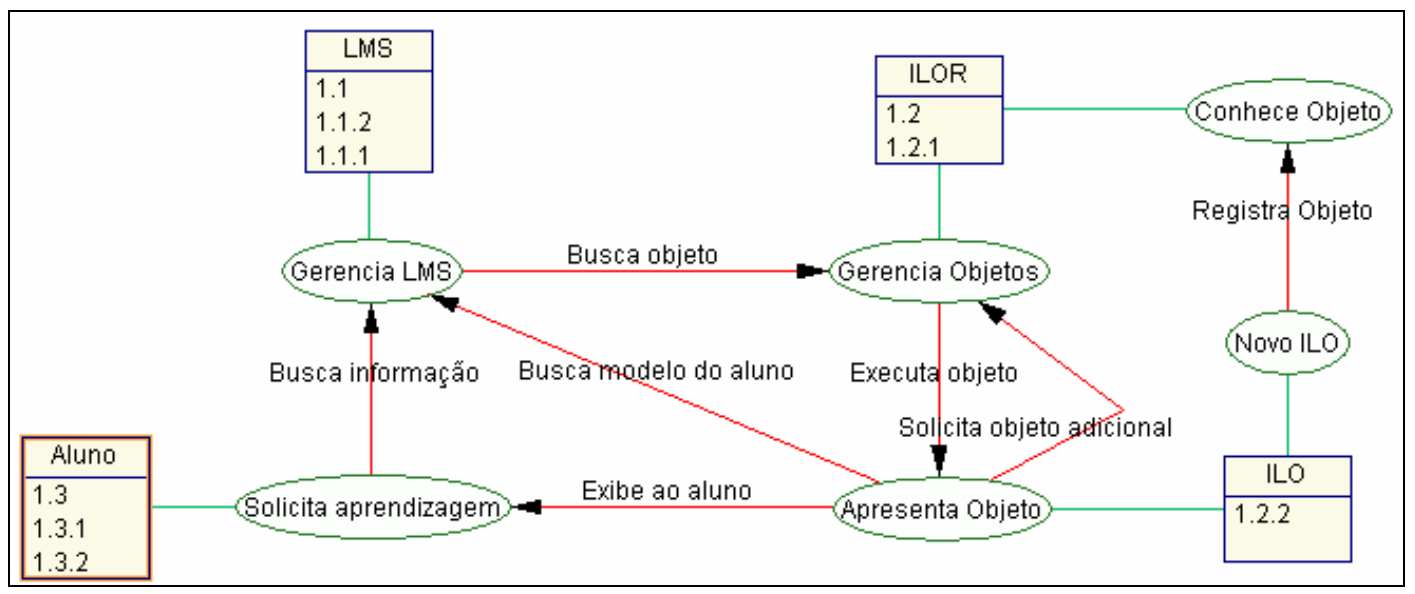

Figura 3. Diagrama de hierarquia de papéis.

Neste trabalho foram identificados quatro papéis que permitirão prover uma experiência de aprendizagem:

- Aluno: gerenciar os dados dos alunos, solicitar nova experiência de aprendizagem e atualizar o modelo do aluno;

- LMS: gerenciar o ambiente, receber solicitação de experiências de aprendizagem, buscar informações sobre o aluno;

- ILOR: gerenciar os objetos de aprendizagem (em seu repositório) e bscar um objeto de aprendizagem; e,

- ILO: apresentar o conteúdo do objeto selecionado ao aluno.

Tais papéis permitirão que as seguintes tarefas sejam desenvolvidas, utilizando um determinado protocolo:

- Solicita experiência de aprendizagem: informa ao LMS que deseja aprender algo; 
- Gerencia o LMS: busca novos objetos para disponibilizar ao aluno;

- Gerencia os Objetos: solicita que um objeto seja executado;

- Apresenta o Objeto: apresenta o objeto ao aluno, e caso a experiência de aprendizagem necessite de complemento com o uso de outros objetos, os solicita;

- Regista o ILO: apresenta-se ao repositório como um novo objeto; e,

- Conhece o objeto: carrega os metadados do objeto no ambiente.

\subsection{Desenvolvendo as Classes dos Agentes}

As classes de agentes são identificadas a partir dos papéis, representadas em caixas, e os diálogos, em linhas, conectando as classes. O diagrama das classes de agentes para Objetos Inteligentes de Aprendizagem é mostrado na Figura 4.

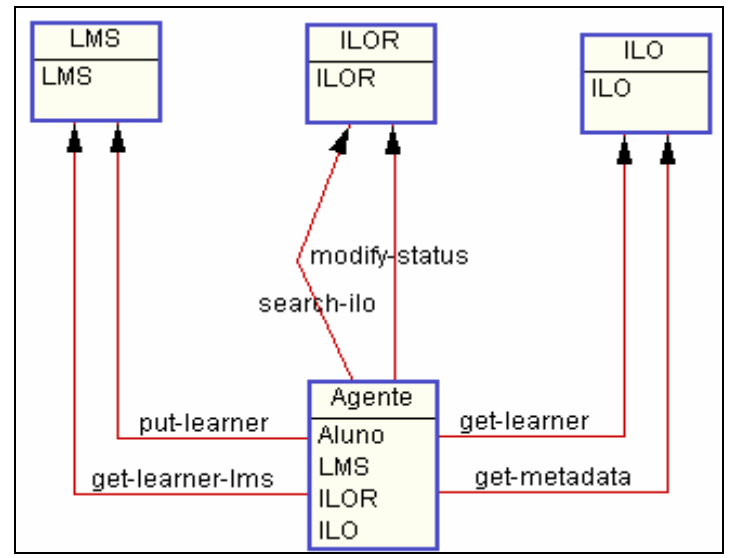

Figura 4. Diagrama de classes de agentes.

Estabeleceram-se três classes de agentes que representarão os papéis de agente LMS, agente ILOR e agente ILO, no que diz respeito a retornar uma determinada informação solicitada. Ainda, conforme descrito na sociedade, qualquer agente poderá estabelecer diálogo em busca de alguma ação, desta forma foi desenvolvido um agente genérico que englobará todos os papéis, representando esta generalidade.

\subsection{Diálogos entre agentes}

O diagrama de diálogo entre agentes é formado por um par de estados finitos que define a comunicação entre duas classes de agentes exclusivamente. $\mathrm{O}$ inicializador começa o diálogo enviando a primeira mensagem. O final do diálogo é representado pelo estado finito terminal.

Conforme identificado no diagrama de classes de agentes, existem 6 diálogos referentes a ontologia ilo-ontology, desenvolvida para a aplicação no domínio de Objetos Inteligentes de Aprendizagem. 
Tabela 1. Diálogos da ontologia ilo-ontology.

\begin{tabular}{l|l|l}
\hline Diálogo & Receptor & Descrição \\
\hline get-learner-lms & LMS & Obtém informações sobre um aluno. \\
\hline put-learner & LMS & Solicita o armazenamento das informações de um aluno. \\
\hline search-ilo & ILOR & $\begin{array}{l}\text { Obtém informações sobre ILOs que atendam a um } \\
\text { determinado critério. }\end{array}$ \\
\hline modify-status & ILOR & Altera o status de um ILO para ativo / inativo. \\
\hline get-learner & ILO & $\begin{array}{l}\text { Obtém informações sobre o estudante com o qual o ILO está } \\
\text { trabalhando. }\end{array}$ \\
\hline get-metadata & ILO & Obtém informações de metadados de um ILO. \\
\hline
\end{tabular}

Os diálogos descritos pela ilo-ontology seguem a mesma sequiência de fases e atos performativos. A Figura 5 ilustra o fluxo de mensagens para o diálogo getmetadata.

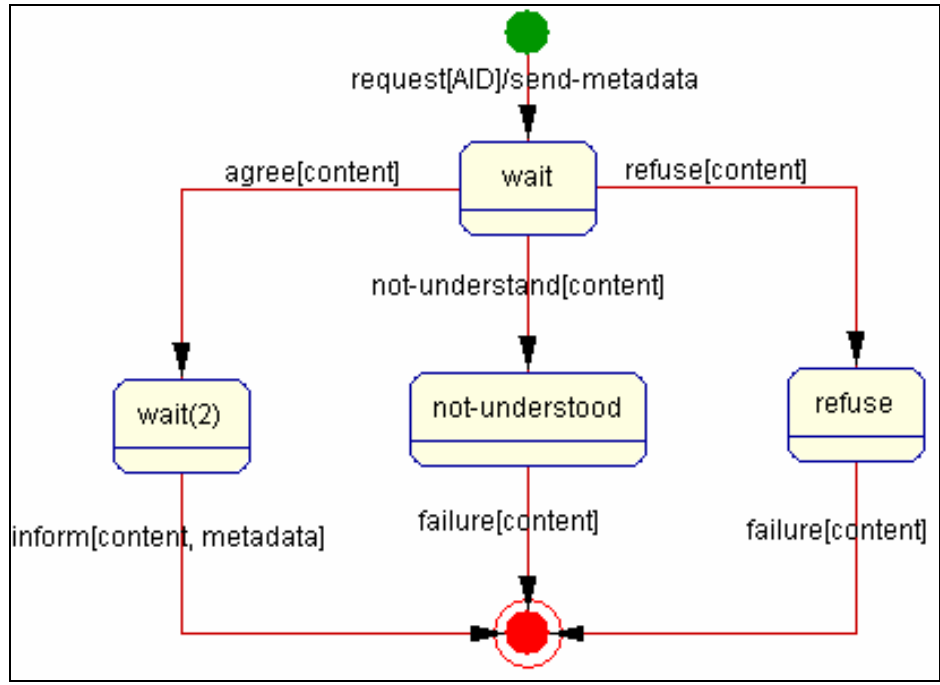

Figura 5. Diagrama do diálogo get-metadata.

O diálogo é composto por três fases: (i) um agente envia uma requisição ao agente ILO informando seu identificador e a ação "send-metadata"; (ii) o agente ILO informa ao agente se aceita, se não compreendeu ou se rejeita a requisição; (iii) o agente ILO retorna a informação solicitada (metadados) ou se o diálogo falhou.

\section{Conclusões}

Os Objetos Inteligentes de Aprendizagem são entidades capazes de promover experiências de aprendizagem através de ambientes de aprendizagem, utilizando como base tecnológica o conceito de objetos de aprendizagem e sistemas multiagentes. Isto, permite a eles interagir com outros agentes, buscando conhecimento sobre o ambiente, alunos e demais objetos que possam auxiliá-lo no ensino de um domínio.

A sociedade proposta por Silveira et al. (2005), foi modelada utilizando uma metodogia própria para sistemas multiagentes, baseada em conceitos da engenharia de software. Esta metodologia facilita o entendimento e possibilita focar o real objetivo do sistema que se deseja construir. Devido a interligação entre os modelos, é possível estabelecer vínculos em todas as etapas, garantindo que todos os objetivos identificados sejam atendidos pelos agentes e diálogos entre eles.

A modelagem de um sistema já proposto permitiu identificar pontos que devem ser modificados ou ampliados. Percebeu-se que os diálogos definidos utilizam apenas 
um processo definido de fases: requisição, confirmação, resposta ou rejeição. A comunicação deve ser revista, envolvendo outros atos performativos, conceitos e ações.

Ainda, verificou-se a necessidade de estabelecer o papel do aluno no ambiente, incluindo-o como um agente que irá interagir com o agente LMS, realizando a solicitação de experiência de aprendizagem. Outro aspecto identificado foi de repassar a tarefa de atualização do modelo do aluno para o agente Aluno, solicitada pelo agente LMS.

Como trabalho futuro, pretende-se avaliar a ontologia proposta por Silveira et al. (2005), na busca de definição mais explícita do conjunto de metadados a serem trabalhados. Serão estudados os modelos de especificação de metadados para Objetos de Aprendizagem existentes a fim de detalhar a ontologia ilo-ontology e, respectivamente, os diálogos entre os agentes.

\section{Referências}

Bradshaw, J. M. (1997). An introduction to software agents In: Bradshaw, J. M. Ed. Software Agents. Massachusetts: MIT Press, 1997.

DeLoach, S. A.; Wood, M. (2000). Developing Muiltiagent Systems with agentTool. In:

Proceedings of Lecture Notes in Artificial Intelligence. Springer - Verlag. Berling, 2001.

Downes, S. (2001). Learning objects: resources for distance education worldwide. In:

International Review of Research in Open and Distance Learning, 2(1). 2001.

IEEE Learning Technology Standards Committee (2004). Specifications. retrieved July 7, 2005 from http://ltsc.ieee.org.

Mohan, P.and Brooks, C. (2003). Engineering a Future for Web-based Learning Objects. Proceedings of International Conference on Web Engineering, Oviedo, Asturias, Spain.

Silveira, R. A., Gomes, E. R, Vicari, R. M. (2005). Inteligent Learning Objects: An Agent-Based Approach of Learning Objects. In Weert, Tom Van, Tatnall, Arthur (Eds.) Information and Communication Technologies and Real-Life Learning. Boston Springer, 1103 - 110.

Sosteric, Mike, Hesmeier, Susan (2002). When is a Learning Object not an Object: A first step towards a theory of learning objects. International Review of Research in Open and Distance Learning. ISSN: 1492-3831 